\title{
Long-Term Experience in Sentinel Node Biopsy for Early Oral and Oropharyngeal Squamous Cell Carcinoma
}

\author{
Kavita M. Pattani, $\mathrm{MD}^{1}$ and Joseph Califano, $\mathrm{MD}^{2,3}$ \\ ${ }^{1}$ Head and Neck Surgical Oncology, MD Anderson Cancer Center Orlando, Orlando, FL; ${ }^{2}$ Department of Otolaryngology- \\ Head and Neck Surgery, Johns Hopkins Medical Institutions, Baltimore, MD; ${ }^{3}$ Milton J. Dance Head and Neck Center, \\ Greater Baltimore Medical Center, Baltimore, MD
}

The presence of nodal disease is a key prognostic indicator in oral cavity and oropharyngeal carcinoma. ${ }^{1,2}$ Detection and management strategies for occult nodal metastasis in oral cavity and oropharyngeal squamous cell carcinoma (OSCC) continue to pose considerable controversy. Clinically negative (cNO) necks in early-stage OSCC tend to harbor occult disease within the cervical lymph nodes in $20-30 \%$ of cases. ${ }^{3-6}$ This high rate of occult nodal metastasis generates substantial clinical interest in optimizing accurate histopathological staging of the neck. The effectiveness of sentinel node biopsy (SNB) has been studied as a potential minimally invasive technique for accurately predicting pathological nodal status. ${ }^{7-11}$

In this study, Broglie et al. prospectively assess the longterm experience and survival data utilizing SNB as a staging tool. Their cohort consisted of 58 patients of whom 29 had a (-) SNB and 29 had a (+) SNB and who were followed by neck dissection. The addition of postoperative radiation was restricted to those patients with $\mathrm{pN} 2 \mathrm{~b} / \mathrm{c}$ disease and (+) ECS only.

Similar to other solid tumor models, including breast, there are efficiencies that are intuitively attractive in performing SNB versus staging neck dissections for OSCC. However, several quandaries still exist in implementing SNB as standard of care for staging patients with OSCC for END. The false-negative rate was reported in this study as $10 \%(3 / 29)$ in those patients with negative SNB. Presumably these patients would have been appropriately staged

\footnotetext{
(C) Society of Surgical Oncology 2011
}

First Received: 14 April 2011;

Published Online: 17 May 2011

J. Califano, MD

e-mail: jcalifa@jhmi.edu and/or treated with selective neck dissection. The authors also indicated that $1 / 29$ patients with SNB (+) had contralateral recurrence, which could indicate a potentially missed sentinel node resulting in persistent disease. Previous studies have reported contralateral sentinel lymph nodes occurring in up to $20 \%$ of cases. ${ }^{12,13}$

Conversely, in the survival analysis performed by Broglie et al., patients exhibited 5 year OS, DFS, and DSS of 88,96 , and $96 \%$ versus 74,73 , and $77 \%$ in (-) SNB compared with $(+)$ SNB, respectively. The DSS in the latter group was statistically significant and demonstrated a persistently poor survival rate in those patients with early stage OSCC and cNO, regardless of having undergone SNB and subsequent END for pathologically node-positive disease. So, although delayed nodal metastases during a waitand-scan policy portend poor overall prognosis, it is unclear if sentinel node biopsy results in any comparable improvement in survival. ${ }^{14-17}$

In view of rising healthcare costs, it is essential to study the survival impact and the cost-effectiveness of performing SNB. Thus, although this study is limited by its cohort size, it would be necessary to demonstrate a survival advantage prior to subjecting patients with a cN0 neck to SNB instead of a wait-and-scan policy. This is especially true if approximately three-quarters of patients (SNB negative) will undergo the procedure with no survival benefit. Moreover, those patients who were identified as the "high-risk" group and selected for END through a $(+)$ SNB still had a lower survival rate yet incurred an additional procedure prior to definitive neck dissection. It is also difficult to define data regarding the surgical complication and morbidities of staging neck dissection compared with sentinel node biopsy.

Although the authors' results are encouraging in demonstrating the feasibility and reliability of SNB, continued 
studies designed in a prospective randomized fashion are still necessary to define further the utility of this method in terms of reducing false-negative SNB and improving survival outcomes. In particular, careful analysis of clinical outcomes and cost benefit of implementing SNB as a standard technique compared with staging neck dissection in select early OSCC is prudent.

ACKNOWLEDGMENT Disclosures Dr. Califano is the Director of Research of the Milton J. Dance Head and Neck Endowment. The terms of this arrangement are being managed by the Johns Hopkins University in accordance with its conflict of interest policies.

\section{REFERENCES}

1. Grandi $\mathrm{C}$, et al. Prognostic significance of lymphatic spread in head and neck carcinomas: therapeutic implications. Head Neck Surg. 1985;8(2):67-73.

2. Kalnins IK, et al. Correlation between prognosis and degree of lymph node involvement in carcinoma of the oral cavity. Am J Surg. 1977;134(4):450-4.

3. Keski-Santti H, et al. Elective neck treatment versus observation in patients with T1/T2 N0 squamous cell carcinoma of oral tongue. Oral Oncol. 2006;42(1):96-101.

4. Pillsbury HC 3rd, Clark M. A rationale for therapy of the N0 neck. Laryngoscope. 1997;107(10):1294-315.

5. Ross GL, et al. Improved staging of cervical metastases in clinically node-negative patients with head and neck squamous cell carcinoma. Ann Surg Oncol. 2004;11(2):213-8.

6. Shah JP, Andersen PE. The impact of patterns of nodal metastasis on modifications of neck dissection. Ann Surg Oncol. 1994;1(6): $521-32$.
7. Paleri V, et al. Sentinel node biopsy in squamous cell cancer of the oral cavity and oral pharynx: a diagnostic meta-analysis. Head Neck. 2005;27(9):739-47.

8. Ross GL, et al. Sentinel node biopsy in head and neck cancer: preliminary results of a multicenter trial. Ann Surg Oncol. 2004;11(7):690-6.

9. Stoeckli SJ. Sentinel node biopsy for oral and oropharyngeal squamous cell carcinoma of the head and neck. Laryngoscope. 2007;117(9):1539-51.

10. Stoeckli SJ, Alkureishi LW, Ross GL. Sentinel node biopsy for early oral and oropharyngeal squamous cell carcinoma. Eur Arch Otorhinolaryngol. 2009;266(6):787-93.

11. Stoeckli SJ, et al. The second international conference on sentinel node biopsy in mucosal head and neck cancer. Ann Surg Oncol. 2005;12(11):919-24.

12. Carlson GW, et al. Management of malignant melanoma of the head and neck using dynamic lymphoscintigraphy and gamma probe-guided sentinel lymph node biopsy. Arch Otolaryngol Head Neck Surg. 2000;126(3):433-7.

13. Wagner JD, et al. Cervical sentinel lymph node biopsy for melanomas of the head and neck and upper thorax. Arch Otolaryngol Head Neck Surg. 2000;126(3):313-21.

14. Borgemeester MC, et al. Ultrasound-guided aspiration cytology for the assessment of the clinically N0 neck: factors influencing its accuracy. Head Neck. 2008;30(11):1505-13.

15. Keski-Santti H, et al. Sentinel lymph node biopsy as an alternative to wait and see policy in patients with small T1 oral cavity squamous cell carcinoma. Acta Otolaryngol. 2008;128(1): 98-102.

16. Sarno A, et al. Does unnecessary elective neck treatment affect the prognosis of N0 laryngeal cancer patients? Acta Otolaryngol. 2004;124(8):980-5.

17. van den Brekel MW, et al. Outcome of observing the N0 neck using ultrasonographic-guided cytology for follow-up. Arch Otolaryngol Head Neck Surg. 1999;125(2):153-6. 